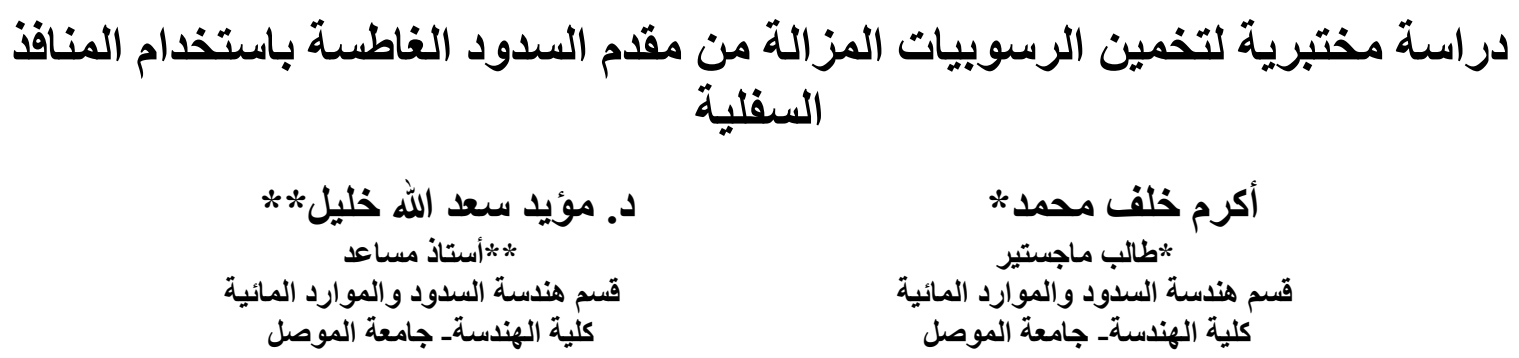

المستخلص

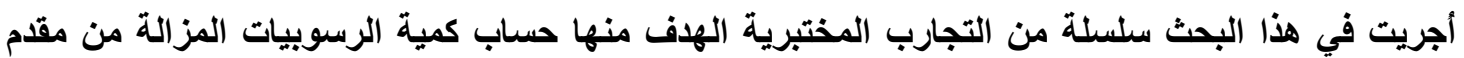

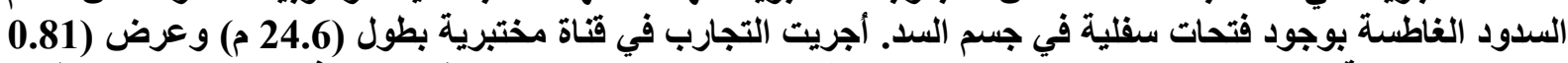

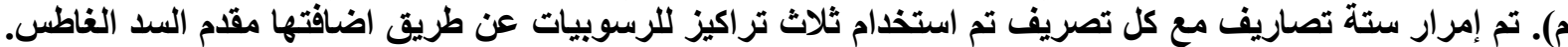

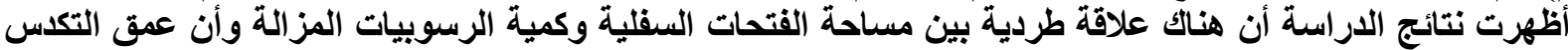

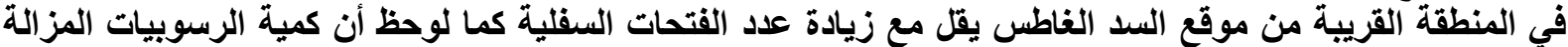

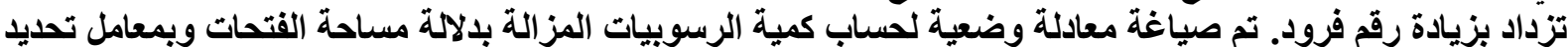

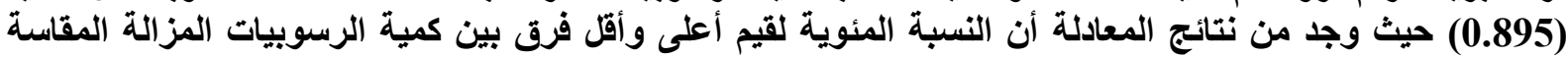

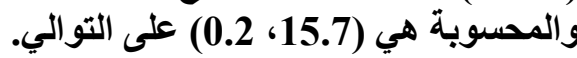

الكلمات الدالة: الرسوبيات المزالة، السدود الغاطسة، المناقذ السفلية.

\title{
Laboratory Study For Estimating The Removing Sediment From Upstream Weirs Using Bottom Outlets
}

\author{
Akram Kh. Mohammed.*
}

Dr. Moayad S. Khaleel.**

\begin{abstract}
In this research, a series of laboratory experiments were carried out to estimate the removing sediment amount from upstream weirs using bottom outlets. The experiments were conducted in a laboratory channel $(24.6 \mathrm{~m})$ in length and $(0.81 \mathrm{~m})$ wide, in middle of which a weir was fixed. Six discharges were allowed to flow with, three different sediment concentration for each. The results showed that there is a direct relation between the area of the holes, Froude number and the amount of sediment removed, the depth of aggradation near weir location decreases with increasing the number of holes. A dimensionless equation was created to compute the amount of sediment removed in terms of area of the holes with a coefficient of determination $(0.895)$ and the percentage of the highest and lowest difference between the measured and calculated sediment amount were $(15.7,0.2)$, respectively.
\end{abstract}


تعد الأنهار أحد المصادر المهمة للماء في الطبيعة ومركز النشاطات البشرية منذ بداية الحضارة. إلا أن هذا

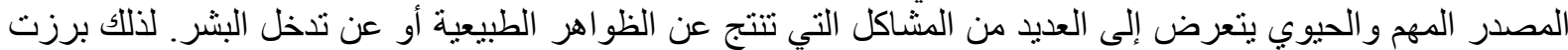

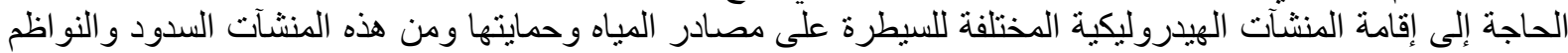

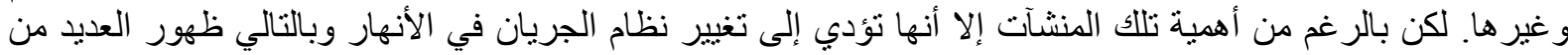

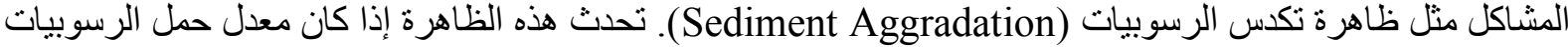

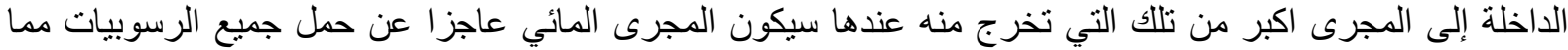

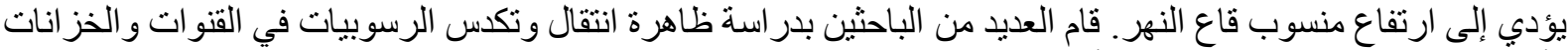

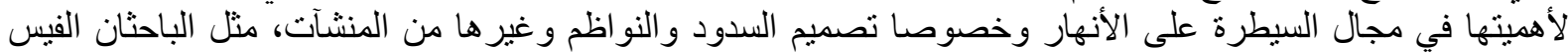

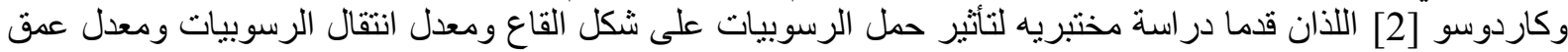

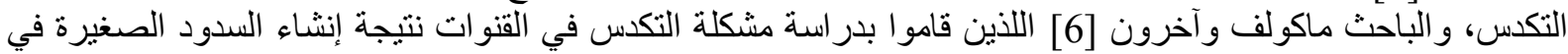

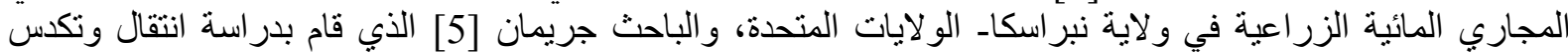

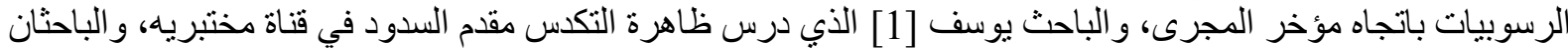

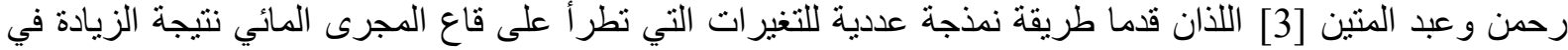

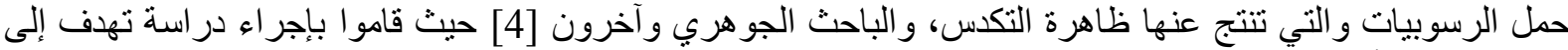

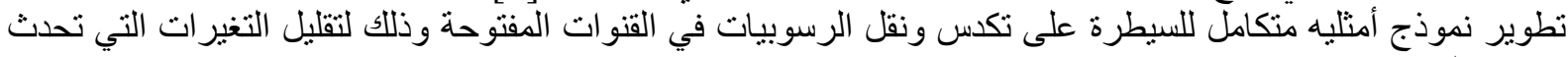
في قاع المجرى.

من الواضح من المصادر العلمية المنوفرة إن استخدام المنشآت الهيدروليكية كالهدارات أو السدود يؤدي إلى إلى تغيير

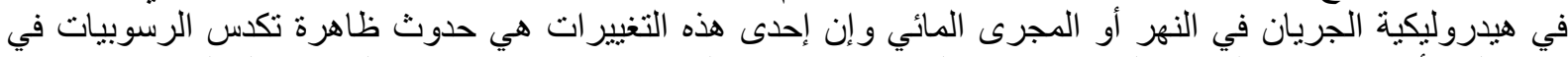

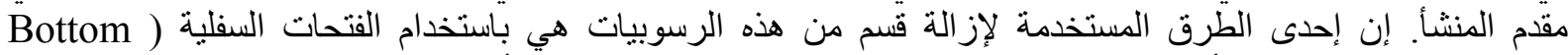
(Outlets

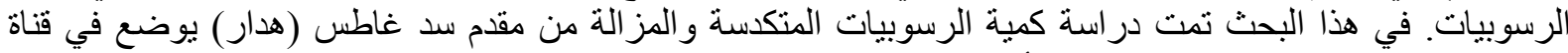

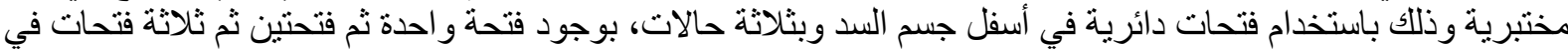

أجريت التجارب في مختبر الهيدروليك_قسم هندسة السدود و الموارد المائية في جامعة الموصل باستخدام قناة

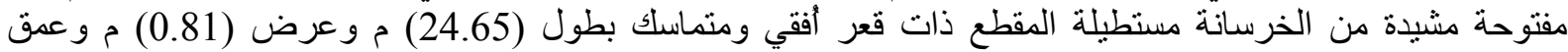

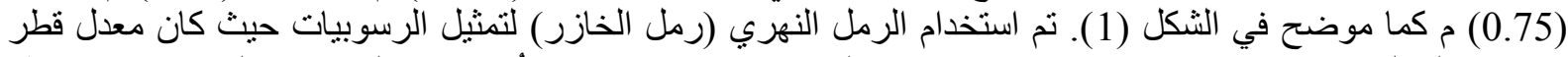
جزيئات الرمل (0) 0.584 mm

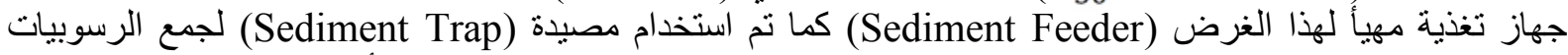

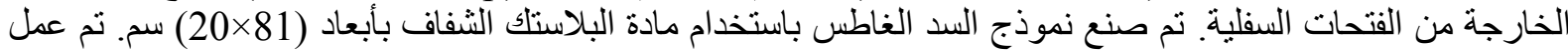
ثلاثة فتحات سفلية في قاعدة السد الغاطس بقطر (24) سم ويمكن غلق ولقد وفتح هذه الفتحات باستخدام سدادة بلاستيكية. تم

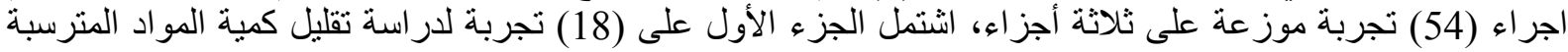

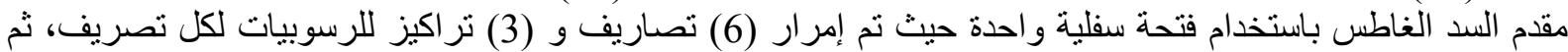

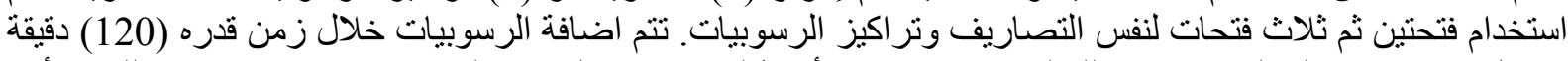

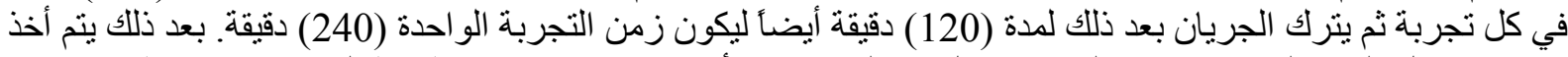

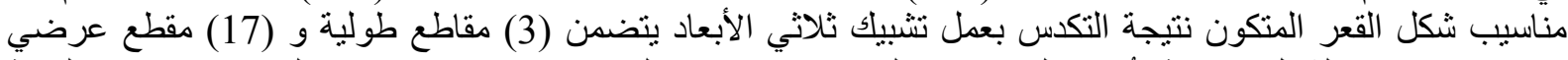

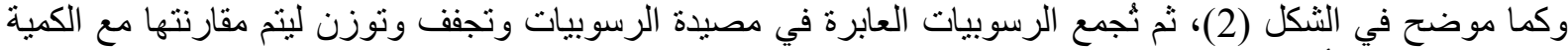

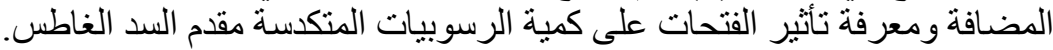


خليل: دراسة مختبرية لتخمين الرسوبيات المزالة من مقدم السدود الغاطسة باستخدام المنافة السفلية

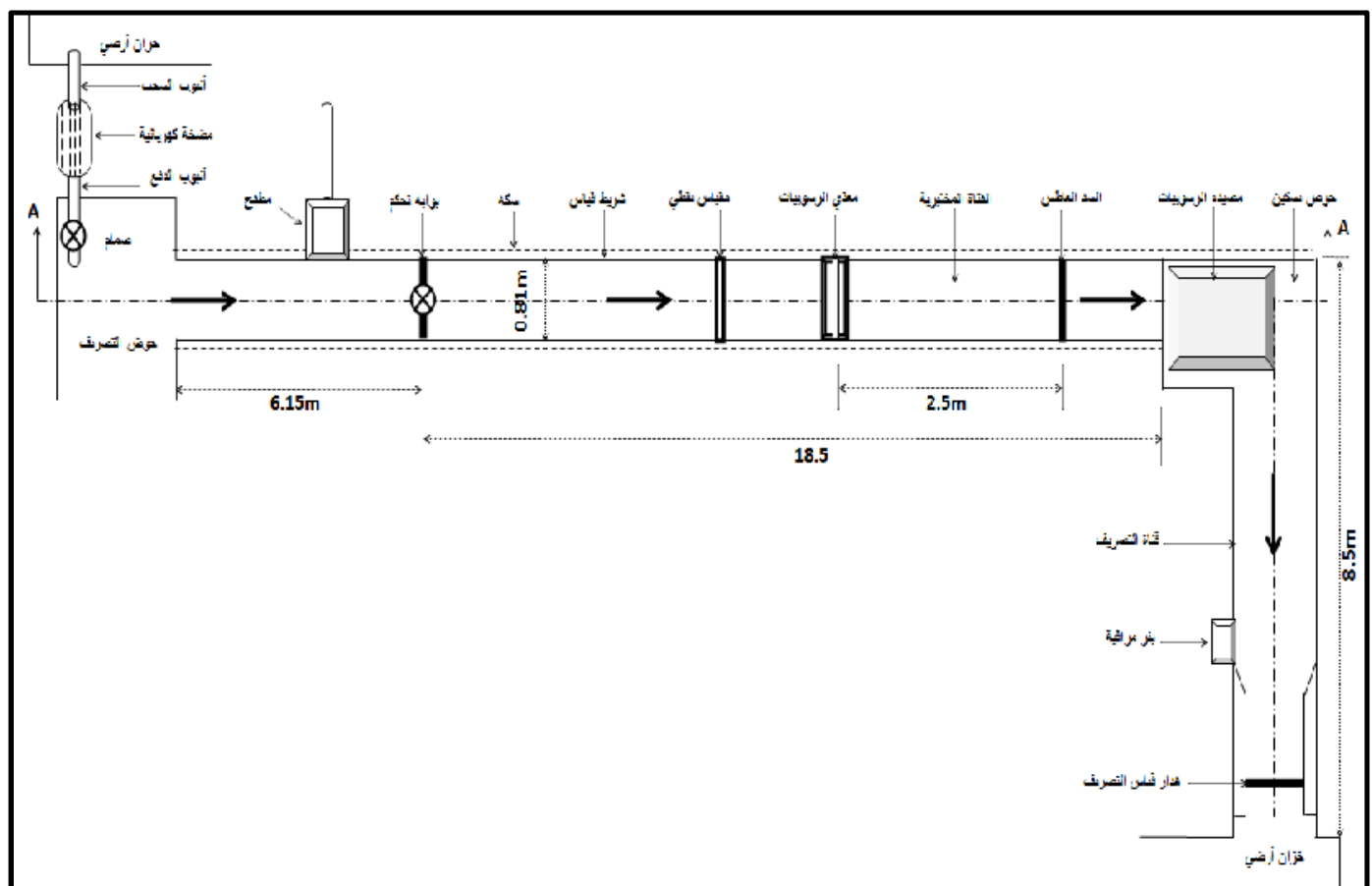

الشكل (1): مخطط القناة المختبرية المستخدمة في الدراسة

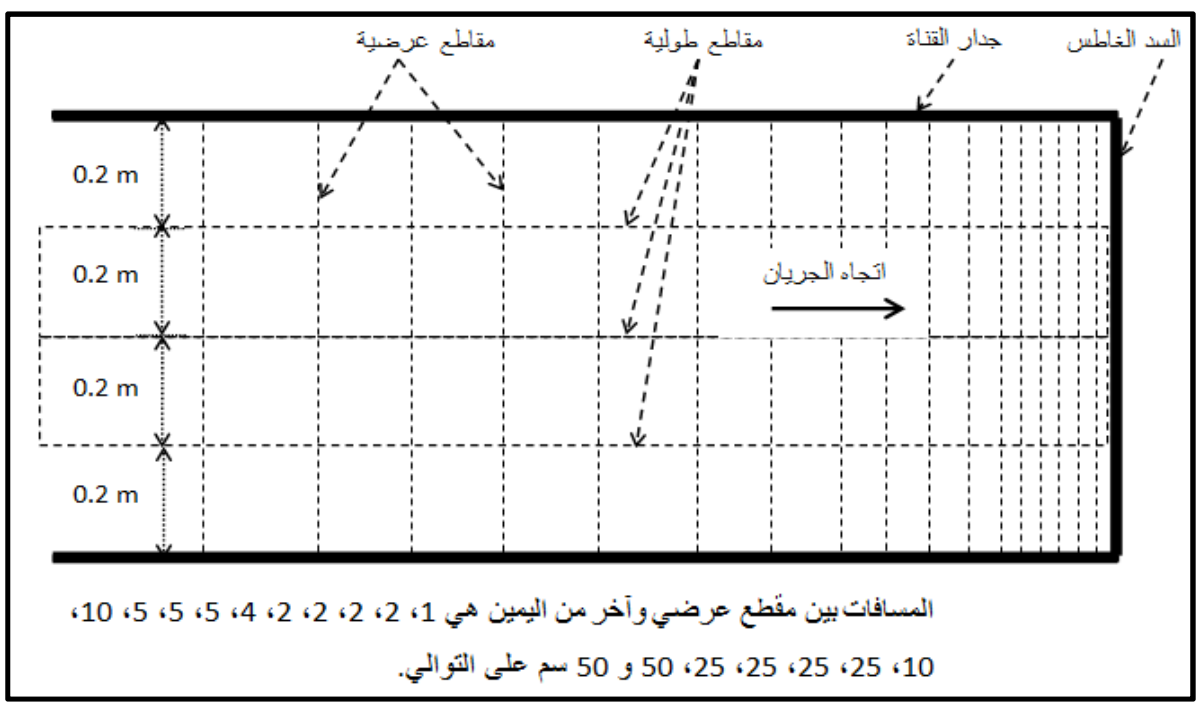

الثكل (2): طريقة التشبيك المستخدمة في تسجيل مناسيب القعر

\section{العوامل المؤثرة على كمية الرسوبيات المزالة عبر المنافن السفلية}

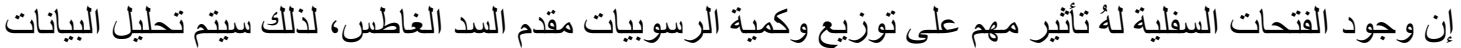

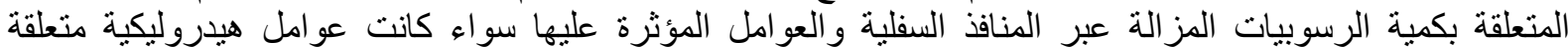

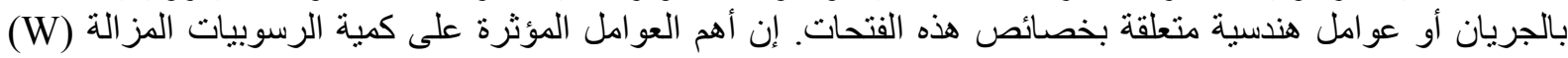


خلال الفتحات السفلية هي التصريف المار في القناة (Q) وتركيز الرسوبيات (C) و عمق الجريان (h) وميل القناة (S)

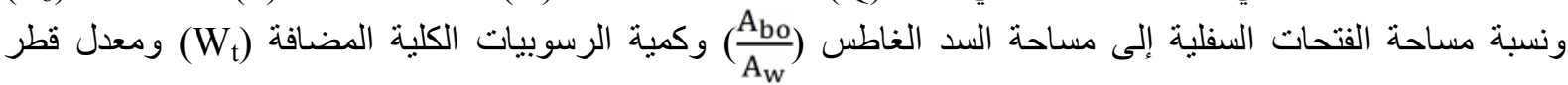

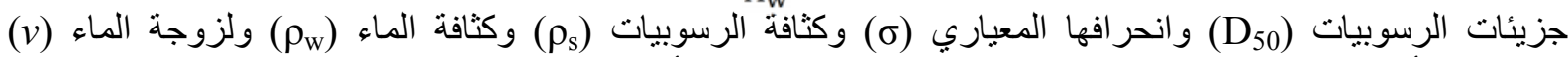
و التعجيل الأرضي (g). حيث نم اجر اء عملية تحليل بعدي للمتغير اتئ أعلات أعلاه وكانت النتيجة كالتالي:

$\frac{\mathrm{W}}{\mathrm{W}_{\mathrm{t}}}=f\left(\mathrm{~F}_{\mathrm{r}}, \frac{\rho_{\mathrm{s}}}{\rho_{\mathrm{w}}}, \operatorname{Re}, \frac{\mathrm{A}_{\mathrm{bo}}}{\mathrm{A}_{\mathrm{w}}}, \sigma, \frac{\mathrm{D}_{50}}{\mathrm{~h}}, \mathrm{~S}_{\mathrm{o}}, \mathrm{C}\right)$

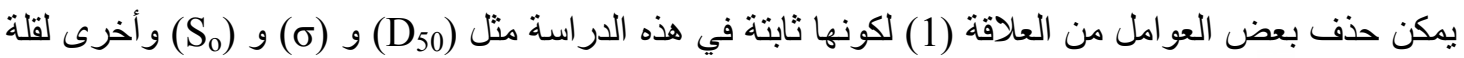
تأثير ها مثل (Re) و (Re $)$ ون $\left(\frac{\rho_{\mathrm{s}}}{\rho_{\mathrm{w}}}\right)$

$\frac{\mathrm{W}}{\mathrm{W}_{\mathrm{t}}}=f_{1}\left(\frac{\mathrm{A}_{\mathrm{bo}}}{\mathrm{A}_{\mathrm{w}}}, \mathrm{F}_{\mathrm{r}}, \mathrm{C}\right)$

\section{النتائَج والمناقشة}

\section{1. ت تأثير مساحة الفتحات السفلية على كمية الرسوبيات المزالة:}

تم تحليل البيانات المختبرية المتعلقة بكمية الرسوبيات المز الة ومساحة الفتحات و المفصلة في الجدول (1) ورئ ورسم

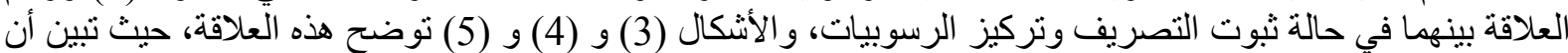

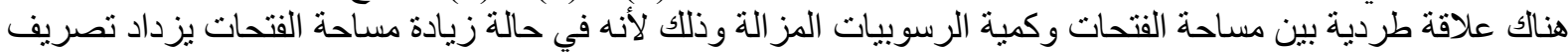

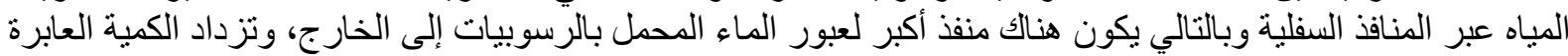
أيضا بزيادة النركيز.

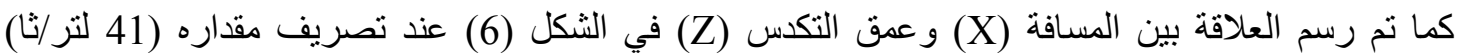

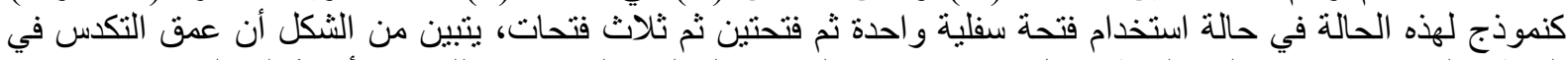
المنطقة القريبة من موقع السد الغاطس يقل مع زيادة عدد الفتحات السفلية والسبب في ذلك فلك يعود أيضاً إلى الزيادة في مساحة منفذ عبور الرسوبيات إلى الخارجة الند الغناطي

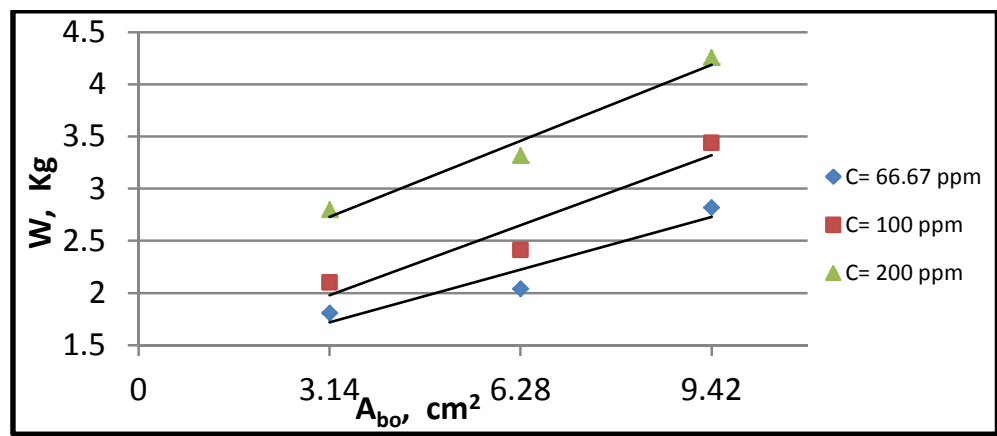

الثكل (3): العلاقة بين مساحة الفتحات وكمية الرسوبيات المز الة للتصريف (30 لتر/ثا). 
خليل: دراسة مختبرية لتخمين الرسوبيات المزالة من مقدم السدود الغاطسة باستخدام المنافذ السفلية

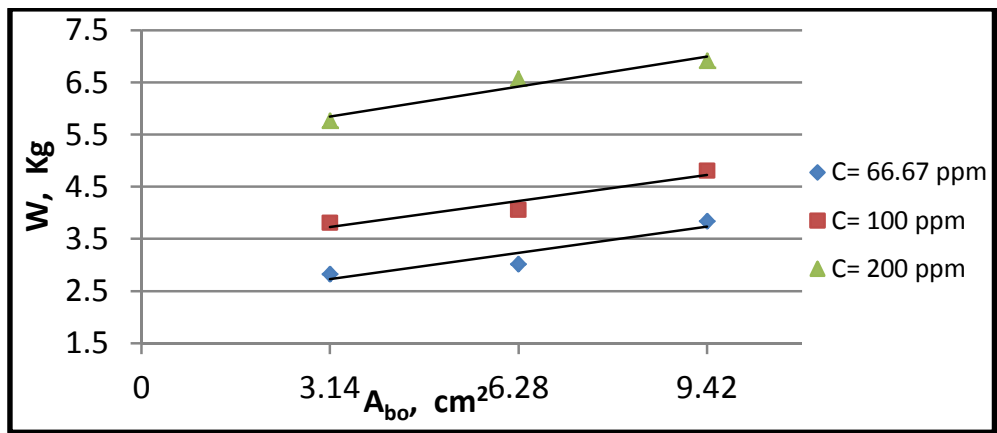

الثكل (4): العلاقة بين مساحة الفتحات وكمية الرسوبيات المز الة للتصريف (41 لتر/ثا).

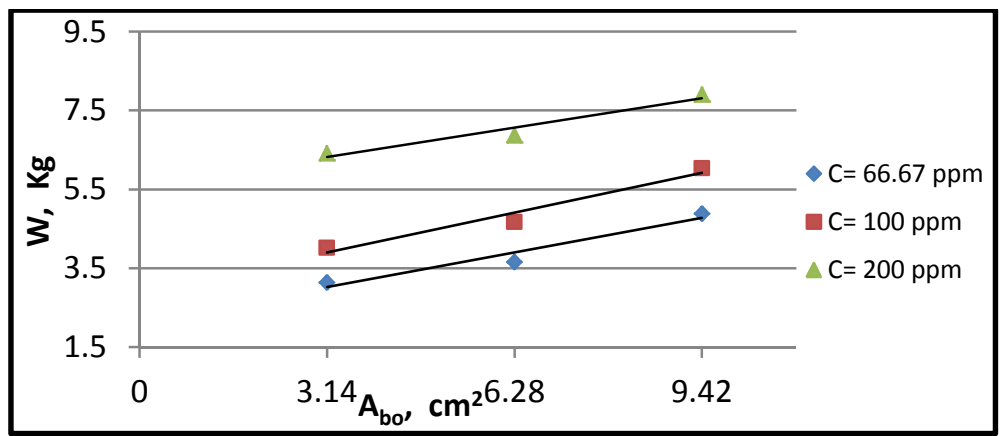

الثكل (5): العلاقة بين مساحة الفتحات وكمية الرسوبيات المز الة للتصريف (50 لتر/ثا).

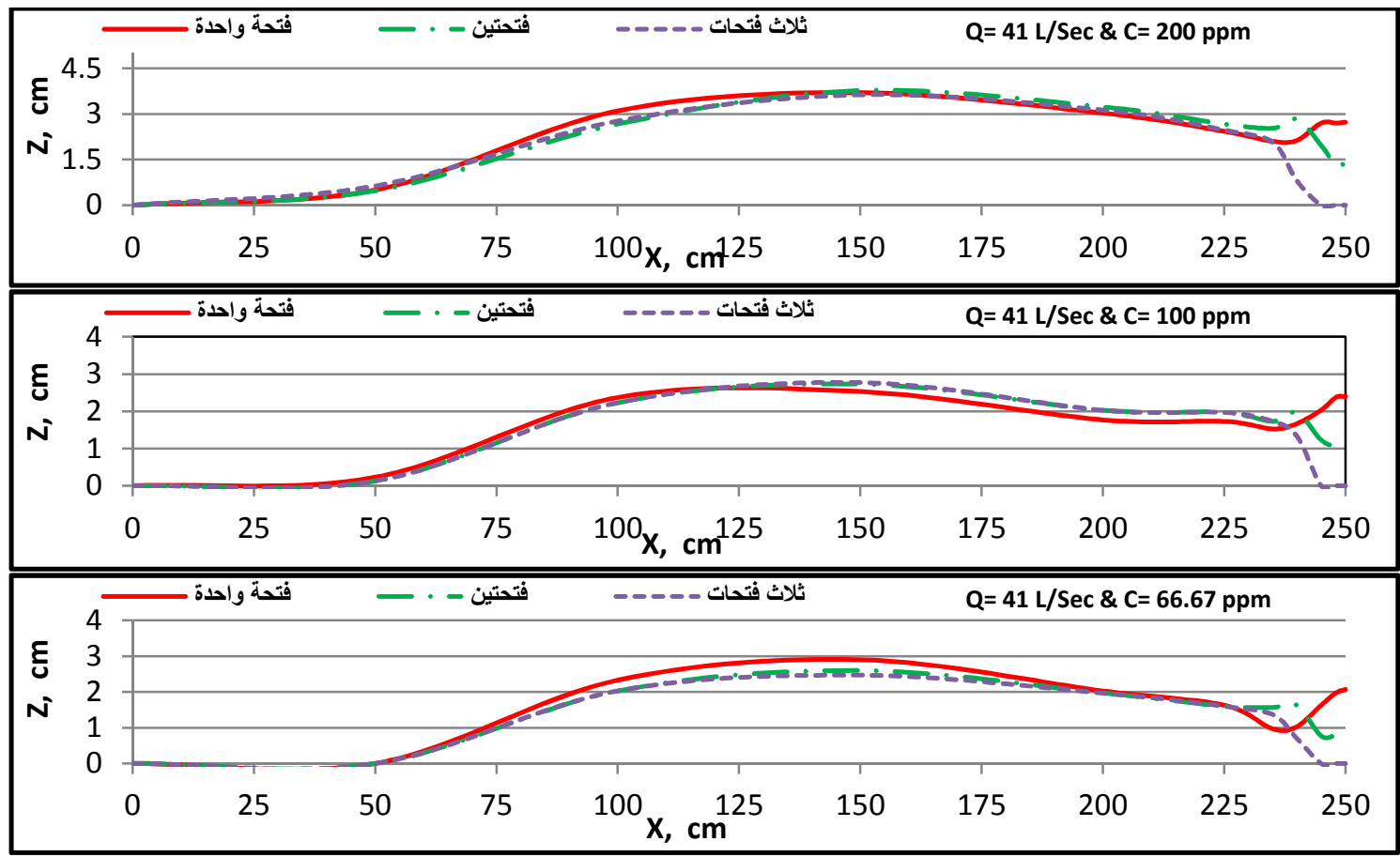

الثكل (6): شكل القعر المتكون عند تصريف (41 لتز/ثا). 
2. تأثير رقم فرود على كمية الرسوبيات المزالة:

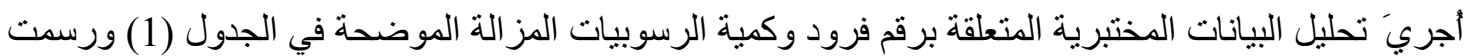

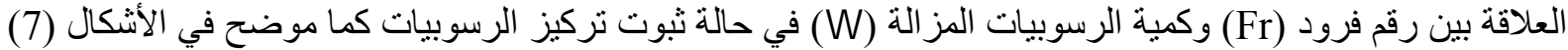

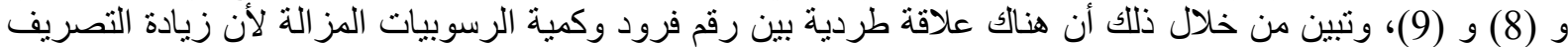

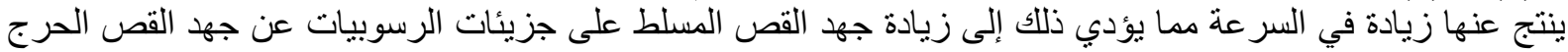

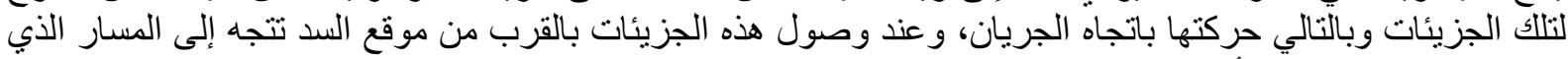

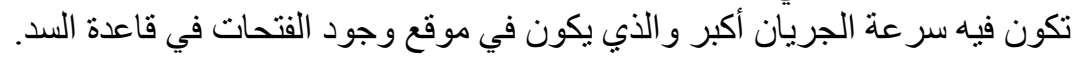
الجدول (1): البيانات المحسوبة من جميع التجارب

\begin{tabular}{|c|c|c|c|c|c|c|}
\hline مساحة الفتحات & الرسوبيات & كفية الرسوبيات & مقدم السدود & التصريف & $\begin{array}{c}\text { التركيز } \\
\text { (C) }\end{array}$ & حالة السد \\
\hline 3.14 & 2.8 & 55.7 & 0.0084 & 30 & \multirow{6}{*}{ 衤 } & \multirow{18}{*}{ 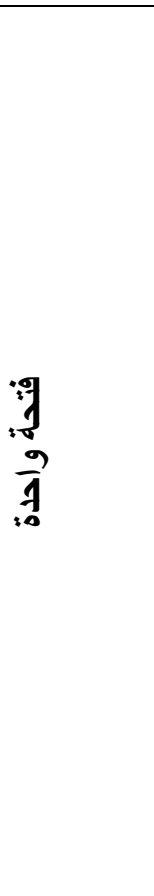 } \\
\hline 3.14 & 3.41 & 62.0 & 0.01058 & 33.4 & & \\
\hline 3.14 & 4.2 & 70.2 & 0.01207 & 37.8 & & \\
\hline 3.14 & 5.77 & 76.2 & 0.0128 & 41 & & \\
\hline 3.14 & 5.89 & 83.6 & 0.0166 & 45 & & \\
\hline 3.14 & 6.42 & 92.9 & 0.017 & 50 & & \\
\hline 3.14 & 2.1 & 27.9 & 0.00853 & 30 & \multirow{6}{*}{$\frac{\Xi}{2}$} & \\
\hline 3.14 & 2.38 & 31.0 & 0.01066 & 33.4 & & \\
\hline 3.14 & 2.76 & 35.1 & 0.0122 & 37.8 & & \\
\hline 3.14 & 3.81 & 38.1 & 0.013 & 41 & & \\
\hline 3.14 & 3.84 & 41.8 & 0.017 & 45 & & \\
\hline 3.14 & 4.02 & 46.4 & 0.0171 & 50 & & \\
\hline 3.14 & 1.81 & 18.6 & 0.0086 & 30 & \multirow{6}{*}{$\begin{array}{l}E \\
\vdots \\
\vdots \\
\vdots \\
0 \\
0 \\
0\end{array}$} & \\
\hline 3.14 & 2.2 & 20.7 & 0.01083 & 33.4 & & \\
\hline 3.14 & 2.5 & 23.4 & 0.01249 & 37.8 & & \\
\hline 3.14 & 2.83 & 25.4 & 0.0131 & 41 & & \\
\hline 3.14 & 2.96 & 27.9 & 0.0171 & 45 & & \\
\hline 3.14 & 3.14 & 31 & 0.0173 & 50 & & \\
\hline 6.28 & 3.32 & 55.7 & 0.0085 & 30 & \multirow{6}{*}{$\frac{\Xi}{2}$} & \multirow{12}{*}{ 诲 } \\
\hline 6.28 & 3.82 & 62.0 & 0.01075 & 33.4 & & \\
\hline 6.28 & 5.58 & 70.2 & 0.01233 & 37.8 & & \\
\hline 6.28 & 6.58 & 76.2 & 0.013 & 41 & & \\
\hline 6.28 & 6.82 & 83.6 & 0.0168 & 45 & & \\
\hline 6.28 & 6.88 & 92.9 & 0.0177 & 50 & & \\
\hline 6.28 & 2.36 & 27.9 & 0.00864 & 30 & \multirow{6}{*}{$\frac{\Xi}{2}$} & \\
\hline 6.28 & 2.64 & 31.0 & 0.01096 & 33.4 & & \\
\hline 6.28 & 3.46 & 35.1 & 0.01244 & 37.8 & & \\
\hline 6.28 & 4.06 & 38.1 & 0.0132 & 41 & & \\
\hline 6.28 & 4.48 & 41.8 & 0.0171 & 45 & & \\
\hline 6.28 & 4.66 & 46.4 & 0.0174 & 50 & & \\
\hline
\end{tabular}


خليل: دراسة مختبرية لتخمين الرسوبيات المزالة من مقدم السدود الغاطسة باستخدام المنافذ السفلية

الجدول (1): تلتمة

\begin{tabular}{|c|c|c|c|c|c|c|}
\hline 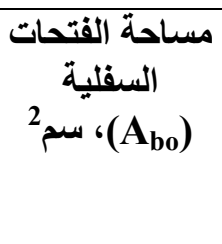 & 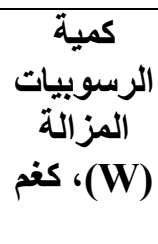 & كغية & رقم فرود السد & $\begin{array}{l}\text { التصريف } \\
\text { لتر/ثاًا، }\end{array}$ & $\begin{array}{c}\text { التركيز } \\
\text { (C) }\end{array}$ & الغاطسة السد \\
\hline 6.28 & 2.04 & 18.6 & 0.00895 & 30 & \multirow{6}{*}{$\begin{array}{l}\vdots \\
\vdots \\
0 \\
0 \\
0 \\
0 \\
0\end{array}$} & \\
\hline 6.28 & 2.28 & 20.7 & 0.01096 & 33.4 & & \\
\hline 6.28 & 2.62 & 23.4 & 0.01273 & 37.8 & & \\
\hline 6.28 & 3.02 & 25.4 & 0.0134 & 41 & & \\
\hline 6.28 & 3.38 & 27.9 & 0.0175 & 45 & & \\
\hline 6.28 & 3.66 & 31 & 0.0179 & 50 & & \\
\hline 9.42 & 4.26 & 55.7 & 0.00975 & 30 & \multirow{12}{*}{$\frac{\Xi}{2}$} & \multirow{18}{*}{ 羿 } \\
\hline 9.42 & 4.12 & 62.0 & 0.01218 & 33.4 & & \\
\hline 9.42 & 6.03 & 70.2 & 0.01339 & 37.8 & & \\
\hline 9.42 & 6.92 & 76.2 & 0.0144 & 41 & & \\
\hline 9.42 & 7.33 & 83.6 & 0.019 & 45 & & \\
\hline 9.42 & 7.91 & 92.9 & 0.021 & 50 & & \\
\hline 9.42 & 3.44 & 27.9 & 0.00951 & 30 & & \\
\hline 9.42 & 3.64 & 31.0 & 0.01188 & 33.4 & & \\
\hline 9.42 & 3.79 & 35.1 & 0.01371 & 37.8 & & \\
\hline 9.42 & 4.81 & 38.1 & 0.0147 & 41 & & \\
\hline 9.42 & 5.09 & 41.8 & 0.019 & 45 & & \\
\hline 9.42 & 6.04 & 46.4 & 0.0197 & 50 & & \\
\hline 9.42 & 2.82 & 18.6 & 0.00975 & 30 & \multirow{6}{*}{$\begin{array}{l}\frac{1}{2} \\
\vdots \\
\hat{2} \\
\dot{0} \\
0\end{array}$} & \\
\hline 9.42 & 2.72 & 20.7 & 0.01218 & 33.4 & & \\
\hline 9.42 & 2.91 & 23.4 & 0.01371 & 37.8 & & \\
\hline 9.42 & 3.84 & 25.4 & 0.0149 & 41 & & \\
\hline 9.42 & 3.71 & 27.9 & 0.0194 & 45 & & \\
\hline 9.42 & 4.89 & 31 & 0.0202 & 50 & & \\
\hline
\end{tabular}

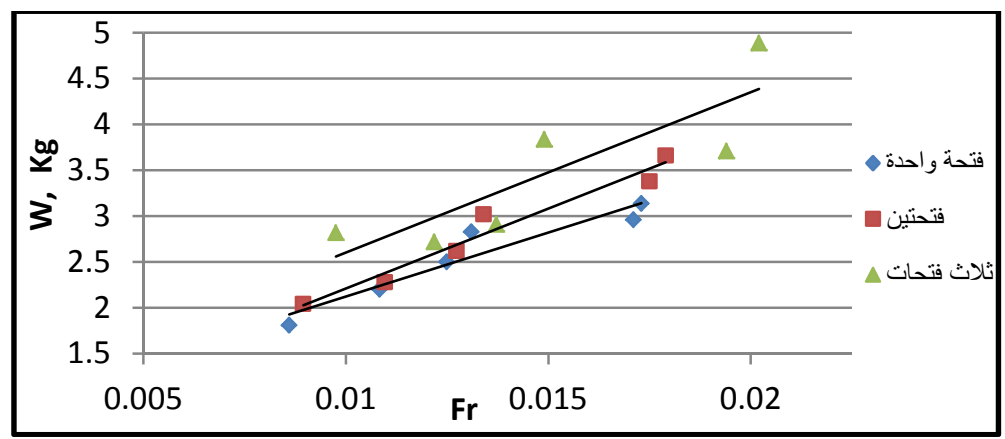

الثكل (7): العلاقة بين رقم فرود وكمية الرسوبيات المز الة للتركيز (66.67 ppm) 


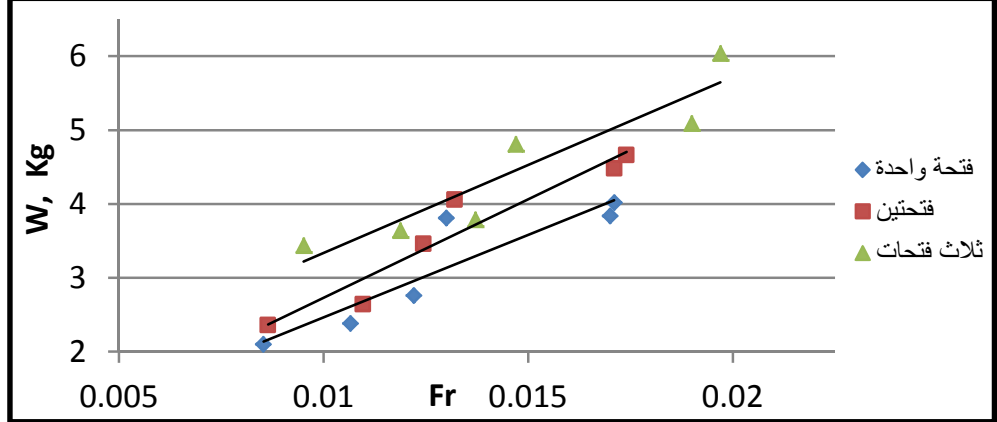

الثكل (8): العلاقة بين رقم فرود وكمية الرسوبيات المز الة للتركيز (100 ppm)

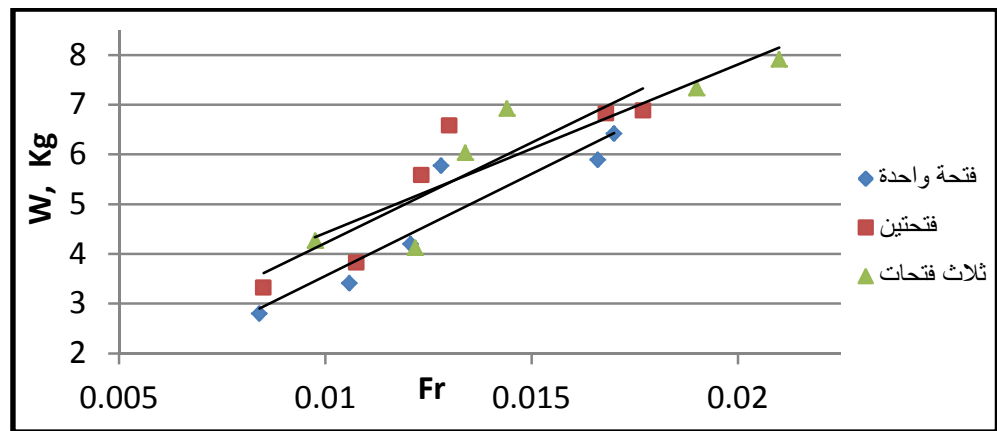

الثكل (9): العلاقة بين رقم فرود وكمية الرسوبيات المز الة للتركيز (200 ppm)

\section{3. ت تأثير تركيز الرسوبيات على كمية الرسوبيات المزالة:}

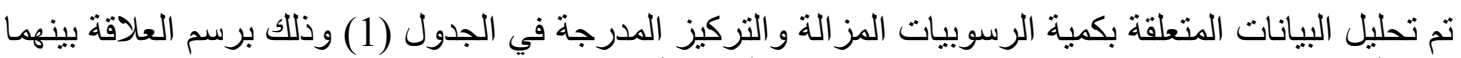

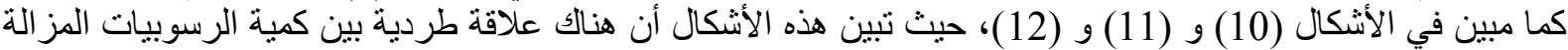

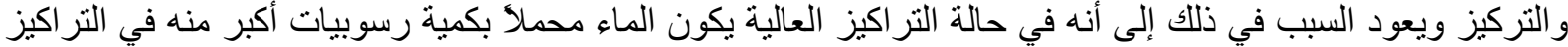
القليلة مما يزيد من الكمية الخارجة عبر الفتحات.

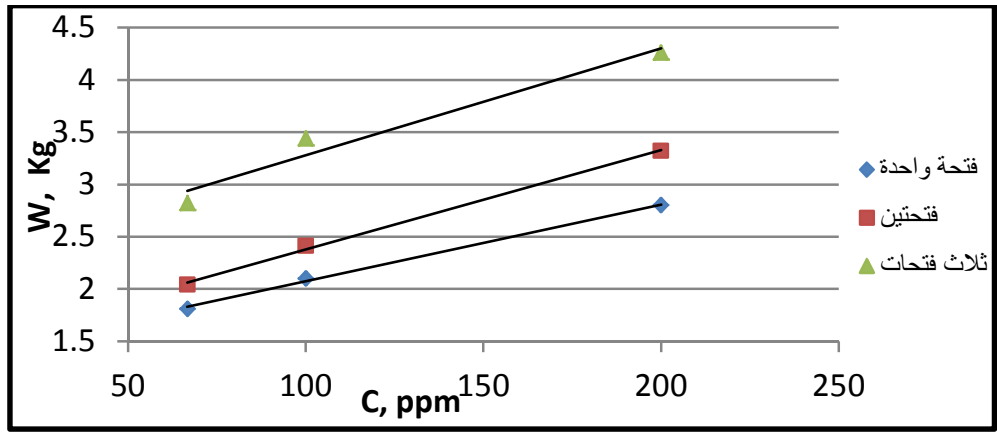

الثكل (10): العلاقة بين التركيز وكمية الرسوبيات المز الة للتصريف (30 لتر/ثا). 


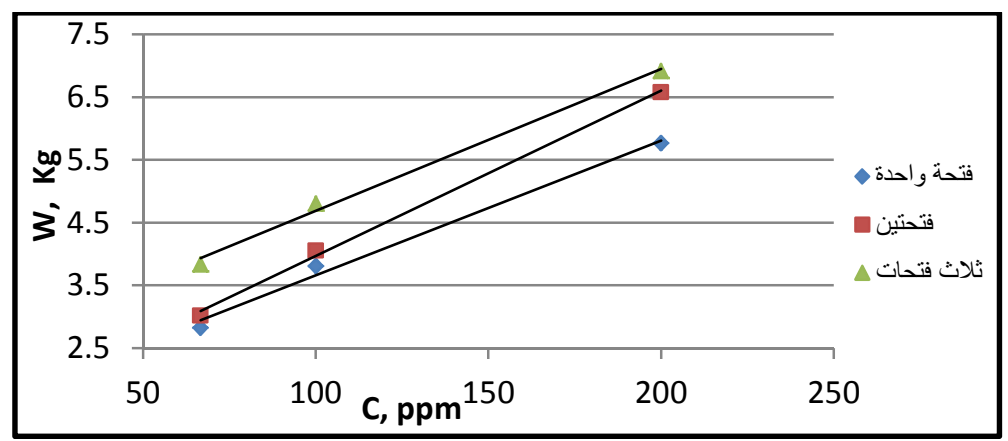

الثكل (11): العلاقة بين التركيز وكمية الرسوبيات المز الة للتصريف (41 لتر/ثا).

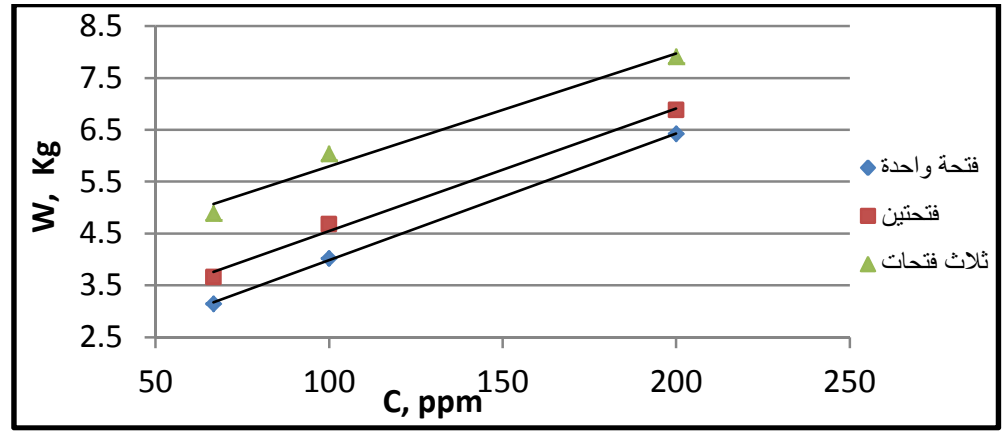

الثكل (12): العلاقة بين التركيز وكمية الرسوبيات المز الة للتصريف (50 لتر/ثا).

\section{صياغة معادلة وضعية لحساب كمية الرسوبيات المزالة}

تم حساب المتغير ات اللابعدية المؤثرة في العلاقة (2) لغرض ايجاد معادلة وضعية تربط بين كمية الرسوبيات

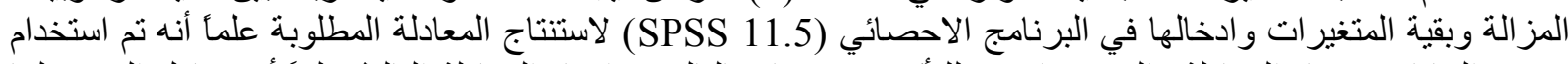

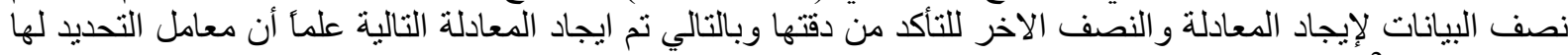
هو

$\frac{\mathrm{W}}{\mathrm{W}_{\mathrm{t}}}=4.6786 \times\left(\frac{\mathrm{A}_{\mathrm{bo}}}{\mathrm{A}_{\mathrm{W}}}\right)^{0.261} \times \mathrm{C}^{-0.462} \times \mathrm{F}_{\mathrm{r}}^{0.218}$

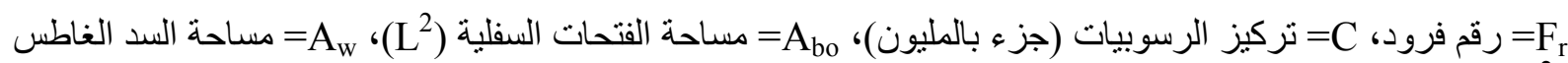

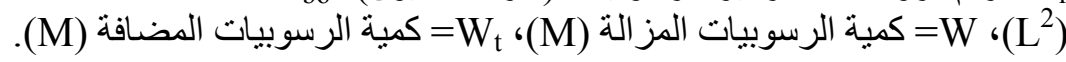

لغرض التحقق من دقة المعادلة (3) تم ادخال البيانات التي لم تستخدم في ايجاد المعادلة وحساب كمية الرسوبيات

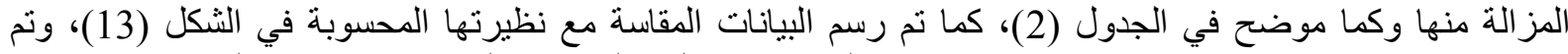

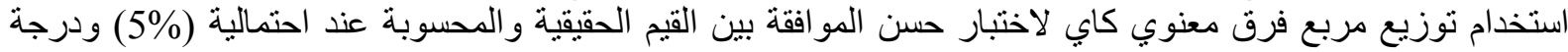

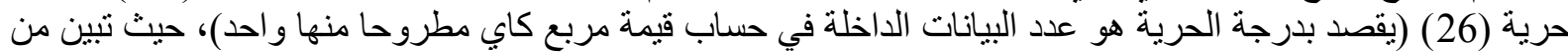

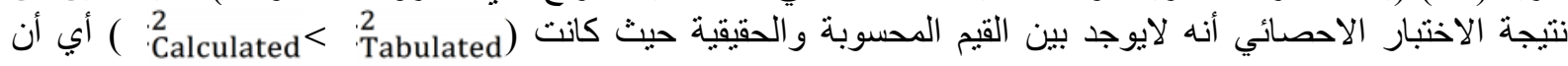




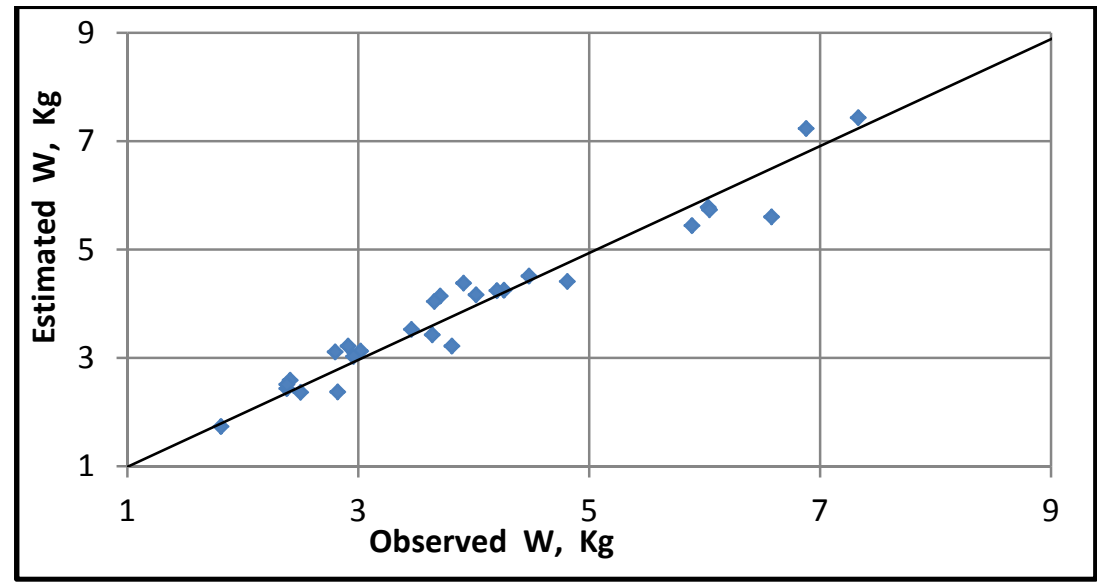

الثكل (13): القيم المقاسة و المحسوبة لكمية الرسوبيات المز الة.

الجدول (2): حساب قيم كمية الرسوبيات المز الة من المعادلة (3) مع نسبة الخطأ

\begin{tabular}{|c|c|c|c|c|c|}
\hline نسبة الخطأ (\% (\% Error & الرسوبياتة المزالة المفم & الرسقاسيةت المزالة المغ & رقم فرود & التركيز(ppm) & $\frac{A_{b o}}{A_{w}}$ \\
\hline 4.2 & 1.734 & 1.81 & 0.0086 & 66.67 & 0.0019 \\
\hline 15.7 & 2.376 & 2.82 & 0.0097 & 66.67 & 0.0058 \\
\hline-7.4 & 2.589 & 2.41 & 0.0086 & 100 & 0.0039 \\
\hline-11.2 & 3.113 & 2.8 & 0.0084 & 200 & 0.0019 \\
\hline 0.2 & 4.250 & 4.26 & 0.0094 & 200 & 0.0058 \\
\hline-2.5 & 2.440 & 2.38 & 0.0110 & 66.67 & 0.0039 \\
\hline-5.6 & 2.513 & 2.38 & 0.0107 & 100 & 0.0019 \\
\hline 5.8 & 3.429 & 3.64 & 0.0119 & 100 & 0.0058 \\
\hline-12.2 & 4.385 & 3.91 & 0.0107 & 200 & 0.0039 \\
\hline 5.3 & 2.368 & 2.5 & 0.0125 & 66.67 & 0.0019 \\
\hline-10.7 & 3.220 & 2.91 & 0.0137 & 66.67 & 0.0058 \\
\hline-1.9 & 3.527 & 3.46 & 0.0124 & 100 & 0.0039 \\
\hline-1.1 & 4.245 & 4.2 & 0.0121 & 200 & 0.0019 \\
\hline 4.0 & 5.786 & 6.03 & 0.0134 & 200 & 0.0058 \\
\hline-3.6 & 3.129 & 3.02 & 0.0134 & 66.67 & 0.0039 \\
\hline 15.4 & 3.224 & 3.81 & 0.0130 & 100 & 0.0019 \\
\hline 8.2 & 4.414 & 4.81 & 0.0147 & 100 & 0.0058 \\
\hline 14.8 & 5.608 & 6.58 & 0.0130 & 200 & 0.0039 \\
\hline-2.4 & 3.031 & 2.96 & 0.0173 & 66.67 & 0.0019 \\
\hline-11.7 & 4.143 & 3.71 & 0.0194 & 66.67 & 0.0058 \\
\hline-0.8 & 4.517 & 4.48 & 0.0174 & 100 & 0.0039 \\
\hline 7.5 & 5.446 & 5.89 & 0.0170 & 200 & 0.0019 \\
\hline-1.4 & 7.435 & 7.33 & 0.0190 & 200 & 0.0058 \\
\hline-10.6 & 4.047 & 3.66 & 0.0175 & 66.67 & 0.0039 \\
\hline-3.7 & 4.170 & 4.02 & 0.0170 & 100 & 0.0019 \\
\hline 5.0 & 5.739 & 6.04 & 0.0197 & 100 & 0.0058 \\
\hline-5.2 & 7.235 & 6.88 & 0.0168 & 200 & 0.0039 \\
\hline
\end{tabular}


من التجارب التي أجريت و النتائج التي تم الحصول عليها وضمن حدود هذه الدر اسة يمكن استنتاج ما يأتي:

1. . توجد علاقة طردية بين مساحة الفتحات السفلية وكمية الرسوبيات المز الة وتزداد شدة العلاقة في حالة زيادة تركيز

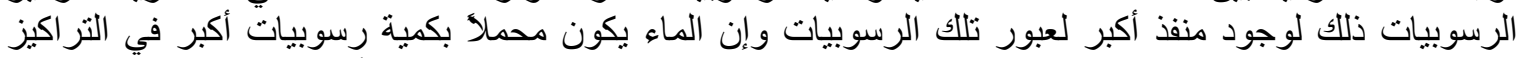

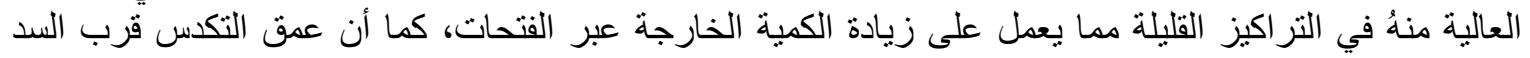

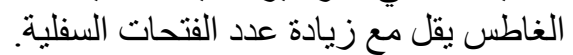

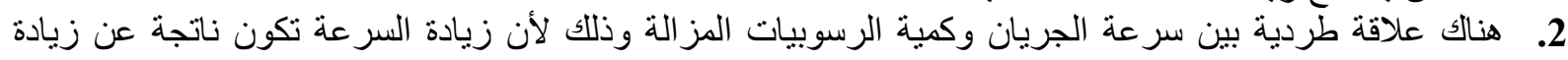

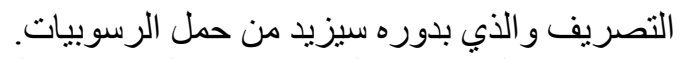

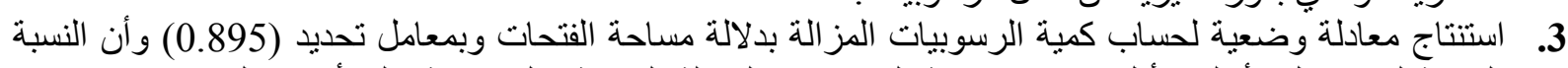

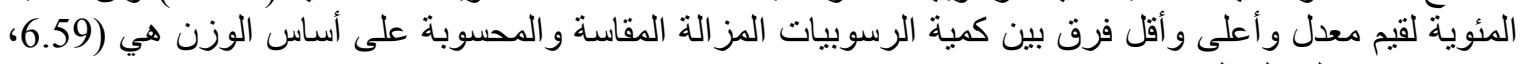

15.7، 15.2 0.2 ) على التوالي.

المصادر العربية

1. يوسف، عبسى الياس عيسى، (2006)، " در اسة مختبرية لخصائص التكدس مقدم السدود"، رسالة ماجستير، قسم هندسة السدود و الموارد المائية، كلية الهندسة، جامعة الموصلة لئل

2. Alves, E., and Cardoso, A. H. (1999), "Experimental Study on Aggradation", International Journal of Sediment Research, Vol. 14, No. 1, pp. 1-15.

3. Ataur, Rahman, Md. and Abdul Matin, Md. (2010), "Numerical Modeling of Bed Level Changes of Alluvial River", Journal of Civil Engineering (IEB), Vol. 38, No. 1, pp. 5364.

4. Elgohry, M., Ding, Y. and Wang, S. S. Y. (2011), "Optimal Sediment Control in Alluvial Rivers", World Environmental and Water Resources Congress 2011: Bearing Knowledge for Sustainability, ASCE 2011.

5. Greimann, B. (2005), "Movement of Sediment Accumulations", Conference of Managing Watersheds for Human and Natural Impacts, 19-22 July, 2005, Williamsburg, Virginia, U.S.A., Published by ASCE, Journal of Water Resources.

6. McCullough, M. C., Harper, J. L., Eisenhauer, D. E., and Dosskey, M. G. (2004), "Channel Aggradation By Beaver Dams on a Small Agricultural Stream in Eastern Nebraska", Conference of The Self-Sustaining Solutions for Streams, Wetlands, and Watersheds, 12-15 September, 2004, Radisson Riverfront Hotel St. Paul, Minnesota, U.S.A., Published by ASAE. 